\title{
Head and Neck Cancer Early Identification of Malnutrition High Risk Patients and Quality of Life Optimization
}

\author{
Joaquim Castro Silva1, Augusta Silveira ${ }^{2,3}$, Ana Sacau², Eurico Monteiro, ${ }^{1,2}$, Teresa Sequeira ${ }^{2,3}$ \\ ${ }^{1}$ Portuguese Institute for Oncology (IPOP), Porto, Portugal \\ ${ }^{2}$ Fernando Pessoa University (UFP), Porto, Portugal \\ ${ }^{3}$ Centre of Health Studies and Research of the University of Coimbra (CEISUC), Coimbra, Portugal \\ Email: j.castrosilva@hotmail.com
}

How to cite this paper: Silva, J.C., Silveira, A., Sacau, A., Monteiro, E. and Sequeira, T. (2019) Head and Neck Cancer Early Identification of Malnutrition High Risk Patients and Quality of Life Optimization. International Journal of Otolaryngology and Head \& Neck Surgery, 8, 204-216.

https://doi.org/10.4236/ijohns.2019.86023

Received: August 19, 2019

Accepted: November 2, 2019

Published: November 5, 2019

Copyright $\odot 2019$ by author(s) and Scientific Research Publishing Inc. This work is licensed under the Creative Commons Attribution International License (CC BY 4.0).

http://creativecommons.org/licenses/by/4.0/

\begin{abstract}
Malnutrition in Head and neck cancer (HNC) patients can be present at the moment of diagnosis. The nutritional status is determinant for the treatment success and quality of life of the patients. The nutritional status gradually declines during treatment and the majority of patients undergoing treatment will need nutritional therapy. On the other hand, HNC, like other cancers, can induce a paraneoplastic syndrome that leads to cachexia. This cachexia status is most of the times the cause of death or the cause of treatment failure. So, early identification of malnutrition high risk patients is crucial to start an adequate nutrition support intervention in HNC patients. This study aims to identify HNC patients who present malnutrition or higher risk of malnutrition; to signalize variables that support early identification of high-risk patients of becoming malnourished and to establish a dynamic relationship between malnutrition risk in these patients and Quality of Life (QoL) impacts. For six months consecutive outpatients with HNC admitted at the Head \& Neck Unity of Oncology Portuguese Institute-Porto were asked to participate in the research $(n=114)$. The European Organisation for Research and Treatment of Cancer (EORTC) cancer-specific HRQoL questionnaire-QLQ-C30 and Malnutrition Universal Screening Tool, MUST were used. At the moment of first presentation, 32 patients (28.1\%) presented high-risk of malnutrition. HNC patients with oral cavity and oropharynx tumour locations, older, with low literacy or with BMI under 18.5 at the moment of diagnosis, represent a high-risk group. When HNC is considered, a dynamic and bi-directional connection between malnutrition and QoL is observed. A significant ( $\mathrm{p}$ $<0.001)$ difference in the scores of Global health status/QoL according to the malnutrition risk group was found: $62.96,53.33,42.71$ for low, medium and
\end{abstract}


high malnutrition risk respectively. Also, Emotional and social functional scales and all symptom scales-including pain, presented significant differences between high and medium risk of malnutrition patients. Fatigue, pain, insomnia, appetite loss and financial difficulties were domains directly related to high risk of malnutrition patients. Pain scores were significantly higher (43.23) in the high-risk patients when compared to medium risk patients (11.67). Nutrition support should be considered at any stage of the pathway - especially in high risk group-in order to optimize tumour treatment results, reduction of adverse effects of therapy and improving both QoL and survival.

\section{Keywords}

Oncology, Head and Neck Cancer, Body Mass Index Malnutrition, Nutritional Support, Quality of Life, Malnutrition Universal Screening Tool

\section{Introduction}

Head and neck cancer (HNC) accounts for more than 550,000 cases and 380,000 deaths annually [1]. In the United States 63.000 new cases occur every year while in Europe a higher incidence is seen-250.000 new cases per year. HNC squamous cell carcinoma represents $3 \%$ and $6 \%$ of all cancer cases respectively [2] [3] [4].

HNC comprehends several locations: the oral cavity, pharynx, larynx, paranasal sinuses and salivary glands and is necessarily related to malnutrition during the disease process and the treatment [5].

Studies reveal that tumour locations, cancer stage, treatment plan, dietary habits, initial weight and body mass index (BMI), lifestyle factors, alcohol and tobacco consumption, oral health performance, social conditions, financial circumstances and cachexia syndrome can guide HNC patients to malnutrition [6] [7] [8].

Significant weight loss and resultant malnutrition caused by a decrease in food intake are expected in HNC patients undergoing radiotherapy. Additionally, poor oral health and chemotherapy act as co-factors in HNC undernourishment. Still, head and neck surgery may cause swallowing dysfunction, xerostomia and severe dysphagia leading to underfeeding. At diagnosis, 20\% - 67\% HNC patients are malnourished or at high risk of becoming malnourished. The malnutrition prevalence rate of HNC patients overtakes 80\% [9] [10] [11].

This aspect is often associated with weight and muscle loss, decrease functioning, depression and negative impacts in Quality of Life (QoL). HNC patients often present delayed wound-healing, oedema and reduced response to chemotherapy and radiotherapy being the effective curative treatments often unsatisfactory. Malnutrition is still linked to increased risk of infection and post-operative complications. Patients malnourished present higher mortality rates and urgent hospital readmissions with economic implications [5] [6] [8] [12]. 
Early identification of high-risk patients and intervention with nutrition support is crucial in HNC [11].

\subsection{Objectives}

This study aims to:

- Identify HNC patients who present malnutrition or higher risk of malnutrition;

- Signalize variables that support early identification of high-risk patients of becoming malnourished;

- Establish a dynamic relationship between malnutrition risk in HNC patients and QoL impacts.

\subsection{Method}

\section{Ethics}

The study was carried out in compliance with the Helsinki Declaration. The method was previously approved by the local research ethical committee and all HNC patients agreed to participate in the research and gave their informed consent. The data were collected for research purposes as part of the routine evaluation.

\section{Patients}

For six months, between January and June 2015, consecutive outpatients with HNC admitted at the Head \& Neck Unity of Oncology Portuguese InstitutePorto (IPOP) were asked to participate $(\mathrm{n}=114)$.

Socio-demographic data and clinical data-age, gender, education, body mass index (BMI), involuntary weight loss, malnutrition risk (Malnutrition Universal Screening Tool, MUST), and tumour location-were collected from the patient's clinical process and complemented, when needed, in semi structured interviews with patients, proxies or clinical staff. In order to better understand socio-demographic and clinical variables, patients were divided in three groups based on MUST risk (low, medium and high).

\section{Questionnaires and scales}

The European Organisation for Research and Treatment of Cancer (EORTC) cancer-specific HRQoL questionnaire-QLQ-C30, were used (Portuguese validated versions).

Questionnaires were completed immediately before consultation as a part of the routine evaluation. Inclusion criteria were: ability to understand written and spoken Portuguese and provision of written consent and physical presence in IPOP for consultation in Head and Neck Unity in the research days.

MUST risk was assessed using three parameters: current BMI, unintentional weight loss and the presence of any acute disease effect that could compromise nutritional intake for $>5$ days. These parameters are rated 0,1 or 2 as follows: BMI $>20 \mathrm{~kg} / \mathrm{m}^{2}=0$, BMI between 18.5 and $20.0 \mathrm{~kg} / \mathrm{m}^{2}=1$ and BMI $<18.5$ $\mathrm{kg} / \mathrm{m}^{2}=2$; unintentional weight loss in the past $3-6$ months $<5 \%=0$, weight loss between $5 \%$ and $10 \%=1$ and weight loss $>10 \%=2$; acute disease absent $=0$ or acute disease present $=2$. 
The overall risk of malnutrition is defined by the sum of all points allocated to each parameter and is classified as follows: $0=$ low-risk; $1=$ medium-risk; $2=$ high-risk [13].

\section{Analysis Strategies and Statistics}

Completed questionnaires were scored according to EORTC instructions. Healthrelated QoL data were analysed by the Statistical Package for Social Sciences (SPSS), version 17 for Windows. Descriptive data are presented with means, standard deviations, medians, ranges, and proportions as appropriate. The chi-squared test was used for testing relationships between the studied variables and to determine whether if there was a significant difference between the expected frequencies and the observed frequencies in the studied variables.

\subsection{Results}

1) HNC Patients' Characteristics

Sociodemographic and clinical characteristics of the studied sample is presented in Table $1(\mathrm{n}=114)$. Patients were mainly male $(93.9 \%)$ and the majority was

Table 1. Head and neck cancer patients characteristics $(n=114)$.

\begin{tabular}{|c|c|}
\hline Characteristics & n (\%) \\
\hline \multicolumn{2}{|l|}{ Gender } \\
\hline Male & $107(93.9)$ \\
\hline Female & $7(6.1)$ \\
\hline \multicolumn{2}{|l|}{ Age (years) } \\
\hline $18-30$ & $1(0.9)$ \\
\hline $31-45$ & $19(16.7)$ \\
\hline $46-65$ & $72(63.2)$ \\
\hline$>65$ & $22(19.3)$ \\
\hline \multicolumn{2}{|l|}{ MUST risk } \\
\hline Low & $72(63.2)$ \\
\hline Medium & $10(8.8)$ \\
\hline High & $32(28.1)$ \\
\hline \multicolumn{2}{|l|}{ Tumor location } \\
\hline Oral cavity \& oropharynx & $38(33.3)$ \\
\hline Hypopharynx \& larynx & $61(53.5)$ \\
\hline Other & $15(13.2)$ \\
\hline \multicolumn{2}{|l|}{ BMI } \\
\hline$>18.5$ & $102(89.5)$ \\
\hline$<18.5$ & $12(10.5)$ \\
\hline \multicolumn{2}{|l|}{ Education } \\
\hline$\leq 6$ years & $86(75.4)$ \\
\hline$>6$ years & $28(24.6)$ \\
\hline
\end{tabular}


46 - 65 years old (63.2\%), revealed low-risk of malnutrition (63.2\%), a BMI greater than 18.5 (89.5\%) and a low educational level (only $24.6 \%$ completed more than 6 schooling years). Hypopharynx and larynx were found to be the major tumour locations (53.5\%).

2) Early identification of high risk of malnutrition in HNC patients according to age, tumour location, $\mathrm{BMI}$ and education

As depicted in Table 2, we found clear relationship between patient's age and the risk levels of malnutrition: high-risk patients were significantly younger than the low-risk malnutrition group $(\mathrm{p}=0.007)$ and less heterogeneous $(51.94 \pm 6.56$ and $58.94 \pm 12.66$, respectively) presenting a lower age range (from 39 to 64 years old).

An association between tumour location and the risk of malnutrition was identified (Chi-squared $=20.287$ degrees of freedom $=4, p=0.000$ ). Indeed, the high-risk malnutrition group presented greater tumour incidence in oral cavity \& oropharynx (15 cases observed compared to 10.7 expected) and revealed an incidence in hypopharynx and larynx locations as expected (16 cases observed similar to 17.1 expected).

It was found an association between BMI and the risk for malnutrition (Chisquared $=26.884$; degrees of freedom $=2 ; \mathrm{p}=0.000)$. Indeed, all patients but one, presenting a BMI lower than 18.5, belonged to the high-risk group for malnutrition.

A relation between education and the risk for malnutrition was detected (Chi-squared $=6.763$; degrees of freedom $=3 ; \mathrm{p}=0.034)$ as depicted in Table 2 . In fact, higher education (more than 6 schooling years) was associated with a low-risk of malnutrition-this group presented fewer patients than expected with high-risk of malnutrition (ratio 0.63) and more patients than expected in

Table 2. Early identification of MUST risk in HNC patients $(\mathrm{n}=114)$ according to age, tumour location, BMI and education (Chi-squared test was used, ${ }^{\star} \mathrm{p}<0.005 ;{ }^{* *} \mathrm{p}<0.001$; Low, $\mathrm{n}=72$; Medium, $\mathrm{n}=10$; High, $\mathrm{n}=32$ ).

\begin{tabular}{|c|c|c|c|}
\hline MUST risk & Low & Medium & High \\
\hline Age mean $\pm S D(n)$ & $58.94 \pm 12.66(72)$ & $52.20 \pm 11.34(10)$ & $51.94 \pm 6.56(32)^{\star}$ \\
\hline \multicolumn{4}{|l|}{ Tumor location \% (n) } \\
\hline Oral cavity \& oropharynx & $13.16(15)$ & $7.02(8)$ & $13.16(15)^{* *}$ \\
\hline Hypopharynx \& larynx & $37.72(43)$ & $1.75(2)$ & $14.04(16)$ \\
\hline Other & $12.28(14)$ & $0.00(0)$ & $0.88(1)$ \\
\hline \multicolumn{4}{|l|}{ BMI \% (n) } \\
\hline$>18.5$ & $62.28(71)$ & $8.77(10)$ & $18.42(21)$ \\
\hline$<18.5$ & $0.88(1)$ & $0.00(0)$ & $9.65(11)^{\star *}$ \\
\hline \multicolumn{4}{|l|}{ Education \% (n) } \\
\hline Up to 6 years $(n=86)$ & $42.98(49)$ & $8.77(10)^{*}$ & $23.68(27)^{\star}$ \\
\hline More than 6 years $(n=28)$ & $20.18(23)^{*}$ & $0.00(0)$ & $4.39(5)$ \\
\hline
\end{tabular}


the low-risk group of malnutrition (ratio 1.30). On the contrary, the less educated group proved to be more vulnerable to malnutrition risk, having more patients than expected both in the high-risk group (ratio 1.12) and in the medium-risk group of malnutrition (1.33).

\section{3) Malnutrition risk in HNC patients and QoL impacts}

It was found that the risk of malnutrition has consistent impact on QoL (Table 3).

Patients at high-risk of malnutrition revealed a constant and significantly lower mean score in Global health status and in QoL. A median of 42.71 was found for high-risk patients group significantly lower than de median found low or medium risk groups, with 62.96 and 53.33 respectively.

When we look for all functional scales-and therefore worse QoL and functioning-the results are comparable, showing lower scores in the high-risk group of patients.

Similar trend was found when considering all symptoms scales where the higher scores-reflecting greater symptomatology-were always found in this group (high-risk).

When considering the functional scales, no differences were found between medium and low risk patients. But for Role, Emotional and Social Functioning scales, significant differences $(\mathrm{p}<0.001)$ occurred between high and medium patient's risk (median scores of 66.15, 65.63 and 77.08 for high-risk patients and 96.67, 90.00 and 96.67 for medium-risk patients when considering Role, Emotional and Social Functioning scales respectively).

Table 3. Impact of malnutrition (MUST risk) on HNC patient's QoL assessed by EORTC QLQ C-30 (Chi-squared test was used; Low, $\mathrm{n}=72$; Medium, $\mathrm{n}=10$; High, $\mathrm{n}=32$ ).

\begin{tabular}{|c|c|c|c|c|}
\hline & Low & Medium & High & $\mathrm{p}$ \\
\hline Global health status/QoL & $62.96 \pm 23.73$ & $53.33 \pm 23.31$ & $42.71 \pm 24.39$ & 0.001 \\
\hline Physical functioning & $82.72 \pm 16.39$ & $86.67 \pm 7.70$ & $75.00 \pm 17.60$ & 0.014 \\
\hline Role functioning & $89.12 \pm 20.01$ & $96.67 \pm 10.54$ & $66.15 \pm 36.30$ & 0.001 \\
\hline Emotional functioning & $79.17 \pm 17.47$ & $89.17 \pm 13.06$ & $65.63 \pm 26.33$ & 0.001 \\
\hline Cognitive functioning & $91.67 \pm 13.12$ & $90.00 \pm 8.61$ & $79.69 \pm 24.22$ & 0.004 \\
\hline Social functioning & $92.13 \pm 13.98$ & $96.67 \pm 7.03$ & $77.08 \pm 27.35$ & 0.001 \\
\hline Fatigue & $14.35 \pm 15.66$ & $14.44 \pm 20.32$ & $34.72 \pm 25.04$ & 0.001 \\
\hline Nausea and vomiting & $2.32 \pm 7.56$ & $1.67 \pm 5.27$ & $7.81 \pm 16.93$ & 0.052 \\
\hline Pain & $15.75 \pm 23.18$ & $11.67 \pm 13.72$ & $43.23 \pm 29.59$ & 0.001 \\
\hline Dyspnoea & $1.36 \pm 0.64$ & $1.10 \pm 0.32$ & $1.66 \pm 0.90$ & 0.049 \\
\hline Insomnia & $1.76 \pm 0.91$ & $1.70 \pm 1.06$ & $2.56 \pm 1.01$ & 0.001 \\
\hline Appetite loss & $1.39 \pm 0.66$ & $1.50 \pm 0.71$ & $2.09 \pm 1.06$ & 0.001 \\
\hline Constipation & $1.19 \pm 0.55$ & $1.00 \pm 0.00$ & $1.72 \pm 1.05$ & 0.001 \\
\hline Diarrhoea & $1.14 \pm 0.39$ & $1.00 \pm 0.00$ & $1.06 \pm 0.25$ & 0.332 \\
\hline Financial difficulties & $1.29 \pm 0.39$ & $1.60 \pm 1.08$ & $2.13 \pm 0.88$ & 0.001 \\
\hline
\end{tabular}


In symptom scales, scores were always higher in the high risk of malnutrition group, revealing to be significantly different from the low risk of malnutrition group except for Diarrhoea and Nausea and vomiting.

The major associations were found in Fatigue, Pain, Insomnia, Appetite loss and Financial difficulties symptoms $(\mathrm{p}=0.001)$. Moreover, Fatigue, Pain, Insomnia and Constipation symptom's differences also occurred between high and medium risk patients. A particularly significant difference verified was in Fatigue and Pain scales, with median scores for high-risk patients of 34.72 and 43.23 and median scores for low-risk patients of 14.35 and 15.75, respectively. The same differences were encountered for medium-risk patients (medium scores of 14.44 for Fatigue scale and 11.67 for Pain scale).

Just like in the functional scales, when considering the symptom scales, no differences were found between medium and low risk patients.

\section{Discussion}

HNC patient's demographic patterns have been changing over the past 30 yearsthey are more likely to be younger adults in their 40s and 50s who never smoked or had reduced tobacco exposure. It is suggested that $70 \%-90 \%$ of new cancers are oropharyngeal, an evidence of the human papillomavirus (HPV) [14]. The studied sample does not follow such trend since patients are older (63.2\% having 46 - 65 years old) and the major tumour location was not oropharyngeal but hypopharynx and larynx.

These findings may reflect a limitation of our study because patients were included while they have been presented to first time consultation in our department for a consecutively period of 6 months. So, this study population may not reflect the real incidence of HNC in Portugal when we are looking for some demographic data or incidence according to HNC primary sites.

Pharynx, larynx, and oral cavity tumour location is a predictor of greater weight loss. Side effects are related to HNC, mainly in oral cavity and oropharynx tumour locations: dysphagia and odynophagia, oral mucositis, dry mouth, taste loss, trismus, muscles fibrosis, ulcerations, teeth loss, chewing and swallowing difficulties [8] [15] [16] [17].

More than half of the problems influenced by HNC have some connection with nutritional problems. Eating-related problems (oral pain, fit of the dental prostheses, prolonged eating time, reduced eating pleasure, and not being able to eat with family and friends) usually appear early and often even before diagnosis (20\% - 67\% are malnourished or at high risk of becoming malnourished at diagnosis). So, malnutrition high risk in HNC patients can happen at presentation and the nutritional status gradually declines during treatment and most patients undergoing treatment will need nutritional therapy [8] [15] [16] [17].

We found the majority of HNC patients had low risk of malnutrition (63.2\%), a clear contrast to values often described in the literature referring up to $80 \%$ malnourished because of their lifestyle and the risk factors associated with this 
disease [18]. Such difference may be explained by the fact that assessment occurred when patients were admitted at the Head \& Neck Unity, before any treatment [19] [20].

Nutritional assessment supports and institutional awareness, may favour early nutritional intervention on nutritional status [21] [22]. Awareness of this fact is of major importance when establishing a treatment and follow-up schemes in HNC patients.

$\mathrm{HNC}$ are a naturally diverse group of tumours linked by anatomical proximity [4]. HNC patients are commonly associated with low patient health literacy as a result of poor education and socioeconomic status [23] [24]. Indeed, the low patient's literacy level found (only $24.6 \%$ completed more than 6 schooling years) reinforces this idea and adds new challenges. Once more the patient recruiting process used in our study can be a limitation when looking to this data because of the possible bias in the population selected.

Certainly, with an aging population and advances in diagnosis and treatment, the number of patients living with the effects of $\mathrm{HNC}$ and its treatment continue to rise. Thus, literacy and educational support for both patients and caregivers, particularly in areas of survivorship, are important to optimize well-being [25] [26].

BMI should be always considered when analysing HNC patients in order to follow weight loss described during treatments [27]. An association between BMI and survival has been described [28]. The BMI observed, greater than 18.5 for the vast majority of the studied patients $(89.5 \%)$ seems thus to constitute a positive prognostic factor. On the other hand, as said before, the recruiting process used in our study may not represent with accuracy the true HNC patients in Portugal. But in this case we don't see this as a limitation of our study because the purpose of the study was to see the relationship of the malnutrition risk in HNC patients and its impact on QoL.

In the present study, patients at high risk of malnutrition revealed a constant and significantly poor QoL in multiple domains. Nutritional status is a strong predictor for HNC patients' QoL before, during and after treatment. Weight loss and malnutrition conduces to nutritional interventions: oral, enteral and parenteral and immune-enhanced nutrition. Tube feeding, usually an invasive procedure, is associated with extended hospitalization, QoL negative impacts and risk of mortality. On the other hand, oral nutritious supplements may introduce several problems: decrease salivary flow and quality, reduce oral clearances, increase bacteria colonization, risk of oral diseases or oral dehydration. QoL domains, such as: swallowing, taste and smell, sticky saliva, mouth pain, speech problems, discouragement or feeling ill, are commonly affected by nutritional interventions. Percutaneous endoscopic gastrostomy, used when long-term tube feeding is necessary, is associated with improved QoL. Quality of nutritious supplements make all difference: the ideal oral supplement should be ready-to-eat, most resemble to regular food (solid like), chewable, moisten, and easy to swallow [15] 
[29] [30] [31]. Our study shows that patients with high-risk for malnutrition have low scores for Global QoL. So routine QoL evaluation, complementing the usual follow-up schemes, is a good surveillance tool and an eventually a good nutritional indicator.

Emotional and social functioning scales and all symptom scales presented significant differences between high and medium risk of malnutrition patients. Functional impairments and psychological distress are common in HNC (20\% - 60\% of patients have depressive symptomatology two to three months post-diagnosis). In the present study, fatigue, pain, insomnia and appetite loss were domains particularly associated to high risk of malnutrition group. There is a dynamic and reciprocal association between QoL domains (depressive and anxiety symptoms, pain, nausea, fatigue, appetite) and weight loss. Pain is considered a robust predictor of poorer QoL in HNC [7] [32] [33].

In this study we can state that pain is the most limitation related to malnutrition. Our data shows that patients in the high-risk group for malnutrition have poorer scores on Pain scale and the impact only occurs in this group (low and medium risk group of patients have similar scores in Pain scale). Future development of this finding could relate QoL evaluation, pain control and analgesics consumption, with nutritional status.

Lifestyle mediation (nutritional and psychosocial support, physical activity orientation) at the moment of diagnosis has been recognized as an important intervention, helping patients to manage side effects and improve their QoL [5].

HNC patients should be subject to a plan contemplating early identification of malnutrition high risk and nutrition interventions. Comprehensive nutritional assessment, documentation, weight loss follow-up and QoL evaluation should be include since admission. It would contribute to identify different needs during the disease trajectory in order to ensure appropriate interventions. Effective nutritional interventions should ultimately aim to improve QoL [16] [17] [19] [22] [34].

\section{Conclusions}

HNC patients are a recognized high-risk group for malnutrition and the diagnostic criteria used to define patients as malnourished lacks uniformity. Perform pre-treatment nutrition and QoL assessments allows early identification of highrisk patients representing a fundamental step to identify nutrition status at baseline, in order to improve it during and after treatments.

HNC patients with oral cavity and oropharynx tumour locations, older, with low literacy or with BMI under 18.5 at the moment of diagnosis, represent a highrisk group.

When HNC is considered, a dynamic and bi-directional connection between malnutrition and QoL is observed. Emotional and social functional scales and all symptom scales-including pain, presented significant differences between high and medium risk of malnutrition patients. Fatigue, pain, insomnia, appetite loss 
and financial difficulties were domains directly related to high risk of malnutrition patients.

Nutrition support should be considered at any stage of the pathway-especially in high risk group-in order to optimize tumour treatment results, reduction of adverse effects of therapy and improving both QoL and survival.

\section{Conflicts of Interest}

The authors declare no conflicts of interest regarding the publication of this paper.

\section{References}

[1] Fitzmaurice, C., Allen, C., Barber, R.M., Barregard, L., Bhutta, Z.A., Brenner, H., et al. (2017) Global, Regional, and National Cancer Incidence, Mortality, Years of Life Lost, Years Lived with Disability, and Disability-Adjusted Life-Years for 32 Cancer Groups, 1990 to 2015: A Systematic Analysis for the Global Burden of Disease Study. JAMA Oncology, 3, 524-548. https://doi.org/10.1001/jamaoncol.2016.5688

[2] Siegel, R.L., Miller, K.D. and Jemal, A. (2017) Cancer Statistics. CA: A Cancer Journal for Clinicians, 67, 7-30. https://doi.org/10.3322/caac.21387

[3] Gatta, G., Botta, L., Sanchez, M.J., Anderson, L.A., Pierannunzio, D., Licitra, L. and EUROCARE Working Group (2015) Prognoses and Improvement for Head and Neck Cancers Diagnosed in Europe in Early 2000s: The EUROCARE-5 PopulationBased Study. European Journal of Cancer, 51, 2130-2143. https://doi.org/10.1016/j.ejca.2015.07.043

[4] Ferlay, J., Shin, H.R., Bray, F., Forman, D., Mathers, C. and Parkin, D.M. (2010) Estimates of Worldwide Burden of Cancer in 2008: GLOBOCAN 2008. International Journal of Cancer, 127, 2893-2917. https://doi.org/10.1002/ijc.25516

[5] Capozzi, L.C., McNeely, M.L., Lau, H.Y., Reimer, R.A., Giese-Davis, J., Fung, T.S. and Culos-Reed, S.N. (2016) Patient-Reported Outcomes, Body Composition, and Nutrition Status in Patients with Head and Neck Cancer: Results from an Exploratory Randomized Controlled Exercise Trial. Cancer, 122, 1185-200. https://doi.org/10.1002/cncr.29863

[6] Power, L, Mullally, D., Gibney, E.R., Clarke, M., Visser, M., Volkert, D., Bardon, L., de van der Schueren, M.A.E., Corish, C.A. and Manuel Consortium (2018) A Review of the Validity of Malnutrition Screening Tools Used in Older Adults in Community and Healthcare Settings: A Manuel Study. Clinical Nutrition ESPEN, 24 1-13. https://doi.org/10.1016/j.clnesp.2018.02.005

[7] Van Liew, J.R., Brock, R.L., Christensen, A.J., Karnell, L.H., Pagedar, N.A. and Funk, G.F. (2017) Weight Loss after Head and Neck Cancer: A Dynamic Relationship with Depressive Symptoms. Head Neck, 39, 370-379. https://doi.org/10.1002/hed.24601

[8] Kang, W.X., Li, W., Huang, S.G., Dang, Y. and Gao, H. (2016) Effects of Nutritional Intervention in Head and Neck Cancer Patients Undergoing Radiotherapy: A Prospective Randomized Clinical Trial. Molecular and Clinical Oncology, 5, 279-282. https://doi.org/10.3892/mco.2016.943

[9] Citak, E., Tulek, Z. and Uzel, O. (2018) Nutritional Status in Patients with Head and Neck Cancer Undergoing Radiotherapy: A Longitudinal Study. Support Care Cancer, 27, 239-247. https://doi.org/10.1007/s00520-018-4319-6 
[10] El Osta, N., El Osta, L., Lassauzay, C., Ghosn, M., Tubert-Jeannin, S. and Hennequin, M. (2018) Oral Health and Chemotherapy Act as Cofactors in Malnutrition in the Elderly with Other Cancers than Head and Neck Malignancies. Clinical Oral Investigations, 23, 235-243. https://doi.org/10.1007/s00784-018-2430-1

[11] Hashida, N., Shamoto, H., Maeda, K., Wakabayashi, H., Suzuki, M. and Fujii, T. (2017) Rehabilitation and Nutritional Support for Sarcopenic Dysphagia and Tongue Atrophy after Glossectomy: A Case Report. Nutrition, 35, 128-131. https://doi.org/10.1016/j.nut.2016.11.003

[12] Torres, T.B., Pomar, B., García Calvo, S., Castro Lozano, M.A., De la Fuente Salvador, B., Izaola Jaúregui, O., López Gómez, J.J., Gómez Hoyos, E., Vaquero Puertas, C. and De Luis Román, D.A. (2018) Clinical and Economic Implications of Disease-Related Malnutrition in a Surgical Service. Nutricion Hospitalaria, 35, 384-391. https://doi.org/10.20960/nh.1315

[13] Stratton, R.J., Hackston, A., Longmore, D., Dixon, R., Price, S., Stroud, M., King, C. and Elia, M. (2004) Malnutrition in Hospital Outpatients and Inpatients: Prevalence, Concurrent Validity and Ease of Use of the "Malnutrition Universal Screening Tool" ("MUST") for Adults. British Journal of Nutrition, 92, 799-808. https://doi.org/10.1079/BJN20041258

[14] Young, D., Xiao, C.C., Murphy, B., Moore, M., Fakhry, C. and Day, T.A. (2015) Increase in Head and Neck Cancer in Younger Patients Due to Human Papillomavirus (HPV). Oral Oncology, 51, 727-730. https://doi.org/10.1016/j.oraloncology.2015.03.015

[15] McClelland, S., Andrews, J.Z., Chaudhry, H., Teckie, S. and Goenka, A. (2018) Prophylactic versus Reactive Gastrostomy Tube Placement in Advanced Head and Neck Cancer Treated with Definitive Chemoradiotherapy: A Systematic Review. Oral Oncology, 87, 77-81. https://doi.org/10.1016/j.oraloncology.2018.10.028

[16] Ehrsson, Y.T., Sundberg, K., Laurell, G. and Langius-Eklöf, A. (2015) Head and Neck Cancer Patients' Perceptions of Quality of Life and How It Is Affected by the Disease and Enteral Tube Feeding during Treatment. Upsala Journal of Medical Sciences, 120, 280-289. https://doi.org/10.3109/03009734.2015.1075630

[17] Gellrich, N.C., Handschel, J., Holtmann, H. and Krüskemper, G. (2015) Oral Cancer Malnutrition Impacts Weight and Quality of Life. Nutrients, 7, 2145-2160. https://doi.org/10.3390/nu7042145

[18] Müller-Richter, U., Betz, C., Hartmann, S. and Brands, R.C. (2017) Nutrition Management for Head and Neck Cancer Patients Improves Clinical Outcome and Survival. Nutrition Research, 48, 1-8. https://doi.org/10.1016/j.nutres.2017.08.007

[19] Sequeira, T., Monteiro, E., Carvalho, C. and Silveira, A. (2017) 10-Year Experience: Routine Assessment of Health Related Quality of Life in Head \& Neck Cancer Patients. Global Journal of Otolaryngology, 12, Article ID: 555835.

https://doi.org/10.19080/GJO.2017.12.555835

[20] Dechaphunkul, T., Martin, L., Alberda, C., Olson, K., Baracos, V. and Gramlich, L. (2013) Malnutrition Assessment in Patients with Cancers of the Head and Neck: A Call to Action and Consensus. Critical Reviews in Oncology/ Hematology, 2, 459-476. https://doi.org/10.1016/j.critrevonc.2013.06.003

[21] Kubrak, C., Martin, L., Gramlich, L., Scrimger, R., Jha, N., Debenham, B., Chua, N., Walker, J. and Baracos, V.E. (2019) Prevalence and Prognostic Significance of Malnutrition in Patients with Cancers of the Head and Neck. Clinical Nutrition. https://doi.org/10.1016/j.clnu.2019.03.030

[22] Silveira, A., Monteiro, E. and Sequeira, T. (2018) Head and Neck Cancer: Improving 
Patient-Reported Outcome Measures for Clinical Practice. Current Treatment Options in Oncology, 19, 59. https://doi.org/10.1007/s11864-018-0578-1

[23] Megwalu, U. (2017) Health Literacy in Patients with Head and Neck Cancer: An Understudied Issue. JAMA Otolaryngology_Head \& Neck Surgery, 143, 645-646. https://doi.org/10.1001/jamaoto.2017.0242

[24] Silveira, A.P., Gonçalves, J., Sequeira, T., Ribeiro, C., Lopes, C., Monteiro, E. and Pimentel, F.L. (2011) Geriatric Oncology: Comparing Health Related Quality of Life in Head and Neck Cancer Patients. Head \& Neck Oncology, 3, 3. https://doi.org/10.1186/1758-3284-3-3

[25] Silveira, A., Amaral, S., Castro, A.R., Monteiro, E., Pimentel, F. and Sequeira, T. (2018) Cancer Palliative Care: Technology Support for Quality of Life Assessment of Family Caregivers. Procedia Computer Science, 138, 294-302.

https://creativecommons.org/licenses/by-nc-nd/4.0 https://doi.org/10.1016/j.procs.2018.10.042

[26] Jabbour, J., Milross, C., Sundaresan, P., Ebrahimi, A., Shepherd, H.L., Dhillon, H.M., Morgan, G., Ashford, B., Abdul-Razak, M., Wong, E., Veness, M., Palme, C.E., Froggatt, C., Cohen, R., Ekmejian, R., Tay, J., Roshan, D. and Clark, J.R. (2017) Education and Support Needs in Patients with Head and Neck Cancer: A Multi-Institutional Survey. Cancer, 123, 1949-1957.

https://doi.org/10.1002/cncr.30535

[27] Lønbro, S., Petersen, G.B., Andersen, J.R. and Johansen, J. (2015) Prediction of Critical Weight Loss during Radiation Treatment in Head and Neck Cancer Patients Is Dependent on BMI. Support Care Cancer, 24, 2101-2109.

https://doi.org/10.1007/s00520-015-2999-8

[28] Grossberg, A.J., Chamchod, S., Fuller, C.D., Mohamed, A.S., Heukelom, J., Eichelberger, H., Kantor, M.E., Hutcheson, K.A., Gunn, G.B., Garden, A.S., Frank, S., Phan, J., Beadle, B., Skinner, H.D., Morrison, W.H. and Rosenthal, D.I. (2016) Association of Body Composition with Survival and Locoregional Control of Radiotherapy-Treated Head and Neck Squamous Cell Carcinoma. JAMA Oncology, 2, 782-789. https://doi.org/10.1001/jamaoncol.2015.6339

[29] Trachootham, D., Songkaew, W., Hongsachum, B., Wattana, C., Changkluengdee, N., Karapoch, J., Thirdsuttironnapumi, S., Meennuch, E., Klaitong, C., Sinthusek, T. and Lam-ubol, A. (2015) Nutri-Jelly May Improve Quality of Life and Decrease Tube Feeding Demand in Head and Neck Cancer Patients. Support Care Cancer, 23, 14211430. https://doi.org/10.1007/s00520-014-2488-5

[30] Barrios, R., Tsakos, G., García-Medina, B., Martínez-Lara, I. and Bravo, M. (2014) Oral Health-Related Quality of Life and Malnutrition in Patients Treated for Oral Cancer. Support Care Cancer, 22, 2927-2933. https://doi.org/10.1007/s00520-014-2281-5

[31] Baldwin, C., Spiro, A., Ahern, R. and Emery, P.W. (2012) Oral Nutritional Interventions in Malnourished Patients with Cancer: A Systematic Review and Meta-Analysis. Journal of the National Cancer Institute, 104, 371-385. https://doi.org/10.1093/jnci/djr556

[32] van der Meulen, I.C., May, A.M., Ros, W.J., Oosterom, M., Hordijk, G.J., Koole, R. and de Leeuw, J.R. (2013) One-Year Effect of a Nurse-Led Psychosocial Intervention on Depressive Symptoms in Patients with Head and Neck Cancer: A Randomized Controlled Trial. Oncologist, 18, 336-344. https://doi.org/10.1634/theoncologist.2012-0299

[33] Scharpf, J., Karnell, L.H., Christensen, A.J. and Funk, G.F. (2009) The Role of Pain in Head and Neck Cancer Recurrence and Survivorship. Archives of Otolaryngolo- 
gy-Head and Neck Surgery, 135, 789-794.

https://doi.org/10.1001/archoto.2009.107

[34] Talwar, B., Donnelly, R., Skelly, R. and Donaldson, M. (2016) Nutritional Management in Head and Neck Cancer: United Kingdom National Multidisciplinary Guidelines. The Journal of Laryngology \& Otology, 130, S32-S40.

https://doi.org/10.1017/S0022215116000402 